\title{
Dyssynchrony Assessment with Tissue Doppler Imaging and Regional Volumetric Analysis by 3D Echocardiography Do Not Predict Long-Term Response to Cardiac Resynchronization Therapy
}

\author{
Suman S. Kuppahally, Michael B. Fowler, Randall Vagelos, Paul Wang, Amin Al-Ahmad, \\ Henry Hsia, and David Liang
}

Department of Cardiovascular Medicine, Stanford University Medical Center, 300 Pasteur Drive, Stanford, CA 94305, USA

Correspondence should be addressed to Suman S. Kuppahally, sumans@yahoo.com

Received 18 August 2010; Revised 9 November 2010; Accepted 28 November 2010

Academic Editor: Dirk Westermann

Copyright (C) 2011 Suman S. Kuppahally et al. This is an open access article distributed under the Creative Commons Attribution License, which permits unrestricted use, distribution, and reproduction in any medium, provided the original work is properly cited.

\begin{abstract}
Background. Currently there are no reliable predictors of response to cardiac resynchronization therapy (CRT) before implantation. We compared pre-CRT left ventricular (LV) dyssynchrony by tissue Doppler imaging (TDI) and regional volumetric analysis by 3-dimensional transthoracic echocardiography (3DTTE) in predicting response to CRT. Methods. Thirty-eight patients (79\% nonischemic cardiomyopathy) with symptomatic heart failure who underwent CRT were enrolled. Clinical and echocardiographic responses were defined as improvement in one NYHA class and reduction in LV end-systolic volume by $\geq 15 \%$ respectively. Functional status was assessed by Minnesota Living with Heart Failure questionnaire and 6-minute walk distance. Results. In 33 patients, after CRT for $7.86 \pm 2.27$ months, there were 24 (73\%) clinical and 19 (58\%) echocardiographic responders. Functional parameters, LV dimensions, volumes and synchrony by TDI and 3DTTE improved significantly in responders. There was no difference in the number of responders and nonresponders when cut-off values for dyssynchrony by different measurements validated in other trials were applied. Area under receiver-operating-characteristic curve ranged from 0.4 to 0.6. Conclusion. CRT improves clinical and echocardiographic parameters in patients with systolic heart failure. The dyssynchrony measurements by TDI and 3DTTE are not comparable and are unable to predict response to CRT.
\end{abstract}

\section{Introduction}

Cardiac resynchronization therapy (CRT) improves functional capacity, quality of life, and reduces heart failure (HF) symptoms. CRT alone without ICD was also shown to reduce mortality in patients with severe HF [1]. Based on large clinical trials $[2,3]$, the current recommendations to select patients for CRT focus on electrical dyssynchrony, such as prolonged QRS duration of $>120$ milliseconds [4]. However, $30-40 \%$ of patients with electrical dyssynchrony do not exhibit mechanical dyssynchrony and remain as nonresponders to CRT [5]. Several studies have been done to find an echocardiographic technique that could predict a favorable response to CRT [6-10]. The goal of these studies has been to find a feasible, inexpensive, and reliable method to identify potential responders to CRT before undergoing the surgical procedure.

Mechanical dyssynchrony studied by tissue Doppler imaging (TDI) has been shown to be a reliable predictor of response after CRT in several studies [8, 11-13]. However, the results of the recent trials, including PROSPECT (predictors of response to CRT), which studied 12 different 2D transthoracic (2DTTE) echocardiographic dyssynchrony parameters, did not find any reliable parameters in predicting the response to CRT at 6 months $[14,15]$. Threedimensional transthoracic echocardiography (3DTTE) [1620 ] with regional volumetric analysis is emerging as a new technique and feasible to assess dyssynchrony. Although realtime 3DTTE has been used to assess dyssynchrony, this technique has not been compared to TDI in predicting 
the response to CRT [21]. We conducted this study to compare dyssynchrony measurements by TDI and regional volumetric analysis by 3DTTE and examine if these methods are efficient and reliable in predicting the long-term clinical and echocardiographic response to CRT.

\section{Methods}

We conducted a single-center, prospective, nonrandomized study comprising 38 consecutive patients who underwent CRT at Stanford University, California, as per recommended guidelines. All the patients signed an informed consent form and a health insurance portability and accountability act (HIPAA) form for the study. Patients with mechanical heart valve(s), pacemaker dependence, and those with technically inadequate echocardiographic images were excluded from the study. Patients underwent clinical and echocardiographic assessment prior to CRT and after 6-12 months of CRT. The clinical parameters included New York Heart Association (NYHA) functional class, 6-minute walk distance (MWD) and Minnesota Living with Heart Failure questionnaire score (MNHFQ). These patients were implanted with Medtronic $(n=29)$ (Insync Sentry, Medtronic Inc.) and St. Judes $(n=4)$ pacemaker devices. After biventricular pacemaker placement, A-V optimization was done by Ritter's method to achieve maximal end-diastolic filling duration, followed by post-CRT imaging within 24 hours of implantation. Clinical response was defined as an improvement in NYHA functional class by one class, and echocardiographic response was defined as a reduction in LV end-systolic volume (LVESV) of $\geq 15 \%$ after 6-12 months of CRT.

2.1. Transthoracic 2D Echocardiography. A Philips Sonos 5500, M2428A 2D Ultrasound system with an S3 transducer was used to obtain LV end-systolic and end-diastolic dimensions. Apical 4-chamber view was obtained to calculate LVESV and LV end-diastolic volume (LVEDV) to give a LVEF by Simpson's method. Mitral regurgitation was semiquantitatively assessed by color Doppler across mitral valve and graded as none (0) trace/mild (1), moderate (2), moderately severe (3), and severe (4), respectively [22].

2.2. Tissue Doppler Imaging. Dyssynchrony index by pulsewave TDI was measured as SD of the time from beginning of QRS to the peak systolic velocity in $12 \mathrm{LV}$ segments, 6 basal and 6 mid segments of inferolateral, inferoseptal, anterior, posterior, anteroseptal, and lateral walls with both CRTon and CRT-off modes. TDI parameter was measured in 3 separate heart beats and averaged for each segment. Then SD for the 12 segments was calculated to derive a dyssynchrony index as 2 SD from the mean. An index of $>32 \mathrm{~ms}$ from the mean was considered as significant dyssynchrony [13]. We also calculated the difference in time to peak systolic velocity between the fastest (Tf) and slowest (Ts) of 6 basal LV segments (Tf-Ts) [12] and calculated septal-lateral delay. A score of $\geq 65 \mathrm{~ms}$ was considered as significant dyssynchrony [11].
2.3. Transthoracic 3D Echocardiography. A transthoracic fullvolume acquisition from apical view using Philips iE33 (Andover, MA, USA) with an X3-1 matrix array transducer was performed with CRT-on and off modes. With 2 perpendicular planes through the LV, a 3-D model of the LV was obtained, and it was subdivided into 17 volumetric segments. Sequence analysis generated the time-volume curves showing the time to the point of minimal systolic volume (Tmsv) for each segment. Q-lab program was run to derive the dyssynchrony index as SD of Tmsv of 12 (6 basal and $6 \mathrm{mid}$ ) and 16 ( 6 basal, $6 \mathrm{mid}$, and 4 apical) LV segments. It also provided the SD of Tmsv of the 12 and 16 segments as a percentage of the R-R interval.

\section{Statistics}

Continuous variables are presented as mean $\pm \mathrm{SD}$, and dichotomous data are presented as numbers and percentages. The clinical and echocardiographic parameters in responders and nonresponders at baseline, immediately after CRT and at 6-12 months after CRT were compared using Student's paired and unpaired $t$-tests, respectively. Categorical variables were compared with Chi-square test, and comparisons between the clinical and echocardiographic endpoints with different dyssynchrony parameters were done using analysis of variance (ANOVA). Receiver-operating characteristics curves (ROC) were generated, and the area under the curve represented the ability of the parameter to predict clinical or echocardiographic response. Correlations between different dyssynchrony measurement techniques were examined by Pearson correlation test. A $P$-value of $\leq .05$ was considered statistically significant.

\section{Results}

Of the 38 patients who underwent CRT, 2 patients had failed biventricular pacing, and 3 patients did not return for followup. After a median followup period of 7 months (7.86 \pm 2.27 months) in 33 patients, symptoms improved in 24 (73\%) patients, unchanged in $7(21 \%)$ patients, worsened in one patient after CRT requiring cardiac transplantation, and one died and these were included as nonresponders $(n=9)$. Of the 24 clinical responders, $18(55 \%)$ were also echocardiographic responders, 5 were clinical responders but not echocardiographic responders, and 1 patient had clinical evaluation but did not get followup echocardiogram. One patient had echocardiographic response without clinical response.

The baseline characteristics and echocardiographic measurements pre-CRT and 6-12 months after CRT are shown in Table 1. There was no significant difference in clinical response in males or females and in patients with nonischemic and ischemic CM (77\% versus 57\%, $P=\mathrm{NS})$. There was a trend towards higher echocardiographic response in nonischemic CM than in ischemic CM (68\% versus $29 \%$, $P=.06)$. Both clinical and echocardiographic responders had longer 6-MWD as compared to nonresponders before CRT, and it remained significant after long-term CRT 
TABLE 1: Baseline characteristics and echocardiographic parameters pre-CRT and after long-term CRT.

\begin{tabular}{|c|c|c|c|}
\hline Characteristic & Baseline & After CRT $(6 \mathrm{~m})$ & $P$-value \\
\hline Age (years) & $59.9 \pm 12.5$ & & \\
\hline Gender: males & $67 \%$ & & \\
\hline Etiology: dilated cardiomyopathy & $79 \%$ & & \\
\hline QRS duration (ms) & $161.2 \pm 16.9$ & & \\
\hline \multicolumn{4}{|l|}{ Clinical parameters: } \\
\hline NYHA class $(1 / 2 / 3 / 4)$ & $0 / 7 / 23 / 3$ & $9 / 17 / 5 / 2$ & $<.0005$ \\
\hline MNLWHF score & $49.7 \pm 22.4$ & $30.4 \pm 22.9$ & $<.0005$ \\
\hline 6-minute walk distance (meters) & $428 \pm 69$ & $482 \pm 91$ & $<.0005$ \\
\hline \multicolumn{4}{|l|}{ Echocardiographic parameters: } \\
\hline LV end-diastolic volume (mL) & $245.3 \pm 128.5$ & $192.5 \pm 101.3$ & .006 \\
\hline LV end-systolic volume (mL) & $188.9 \pm 109.1$ & $125.8 \pm 80.3$ & .0002 \\
\hline LV ejection fraction (\%) & $25 \pm 6$ & $38 \pm 11$ & .0001 \\
\hline LV end-diastolic diameter $(\mathrm{cm})$ & $6.9 \pm 1.5$ & $6.3 \pm 1.5$ & .004 \\
\hline LV end-systolic diameter $(\mathrm{cm})$ & $6.0 \pm 1.5$ & $5.1 \pm 1.6$ & .0008 \\
\hline LV fractional shortening (\%) & $13.3 \pm 0.5$ & $19 \pm 0.9$ & .006 \\
\hline Mitral regurgitation & $1.67 \pm 1.1$ & $1.32 \pm 0.8$ & .03 \\
\hline TDI: SD of Tf-Ts in 12 segments (ms) & $44.2 \pm 14.1$ & $34.1 \pm 14.4$ & .0008 \\
\hline TDI: SD of Tf-Ts in 6 basal segments (ms) & $106.3 \pm 41.7$ & $76.5 \pm 35.8$ & .0001 \\
\hline TDI: SD of septal-to-lateral delay (ms) & $58.1 \pm 37.1$ & $61.2 \pm 36.8$ & .32 \\
\hline 3D: SD of Tmsv in 6 basal segments (ms) & $84.6 \pm 56.4$ & $53.9 \pm 41.3$ & .01 \\
\hline 3D: SD of Tmsv in 12 segments (ms) & $46.3 \pm 31.1$ & $24.4 \pm 20.5$ & .002 \\
\hline 3D: SD of Tmsv in 16 segments (ms) & $58.1 \pm 35.2$ & $35 \pm 28.8$ & .04 \\
\hline 3D: SD of Tmsv in 12 segments as \% of R-R interval & $5.8 \pm 4.2 \%$ & $3.1 \pm 2.9 \%$ & .0004 \\
\hline 3D: SD of Tmsv in 16 segments as \% of R-R interval & $7.0 \pm 4.1 \%$ & $4.3 \pm 3.8 \%$ & .01 \\
\hline
\end{tabular}

CRT: cardiac resynchronization therapy, LV: left ventricle, TDI: tissue Doppler imaging, Tf-Ts: difference in time to peak systolic velocity between the fastest and slowest LV segments, Tmsv: difference in longest and shortest time to minimal systolic volume in LV segments, NYHA: New York Heart Association, MNHFQ: Minnesota Living with Heart Failure questionnaire, SD: standard deviation.

(Table 1). The pre-CRT HF medications, such as betablockers, ACEI/ARB, diuretics, and aldosterone receptor antagonists remained the same after CRT without any significant change in their doses.

LV pacing lead was placed in posterior or postero-lateral position $(n=24)$, antero-lateral position $(n=3)$ patients, middle-cardiac-vein $(n=4)$ patients, and epicardially placed in 2 patients. There was no difference in the lead position in the clinical or echocardiographic responders and nonresponders.

4.1. Immediate Response to CRT. After initiation of CRT and $\mathrm{AV}$ optimization, there was no change in clinical symptoms, but there was a significant reduction in LV dyssynchrony indices measured by TDI $(44.2 \pm 14.1$ versus $33.5 \pm 13.5$, $P<.05$ in $12 \mathrm{LV}$ segments) and 3DTTE $(46.2 \pm 31.0$ versus $28.3 \pm 19.0, P<.05$ in $12 \mathrm{LV}$ segments). Analysis of the SD of Tmsv as a function of the R-R interval in 12 segments $(5.8 \pm 4.2 \%$ versus $4.5 \pm 3.7 \%, P=\mathrm{NS})$ and 16 segments $(7.0 \pm 4.1 \%$ versus $5.6 \pm 4.2 \%, P=\mathrm{NS}$ ) by 3 DTTE did not show significant improvement. The immediate reduction in dyssynchrony score by TDI and 3DTTE did not correlate with the clinical or echocardiographic response at 6-12 months.
4.2. Predictors of Clinical Response. After CRT of $7.86 \pm 2.27$ months, $24(73 \%)$ were clinical responders with improvement in 1 NYHA functional class. In these patients, there was a significant improvement in $6-\mathrm{MWD}(\Delta 71 \mathrm{~m}$ versus $\Delta 22 \mathrm{~m}$, $P<.05)$ and in MNHFQ score $(\Delta 22$ versus $\Delta 12, P<.05)$ as compared to nonresponders.

Clinical responders were identified with $75 \%$ sensitivity and $38 \%$ specificity $(\mathrm{ROC}=0.69)$ with pre-CRT QRS duration of $150 \mathrm{~ms}$. Dyssynchrony score of $32 \mathrm{~ms}$ with TDI identified responders with a sensitivity of $75 \%$ and a specificity of $12 \%$. The clinical response was not significantly different in those who met these cut-off values as compared to those who did not (Table 2). Echocardiographic improvement in LVESV $>15 \%$ identified clinical responders (ROC $=$ $0.88, P<.003$ ) with $84 \%$ sensitivity and $86 \%$ specificity. PreCRT LVESV did not predict clinical response. There was no significant difference in LV dyssynchrony delta change values in clinical responders and nonresponders (Table 2).

4.3. Predictors of Echocardiographic Response. After longterm CRT, the average LVESV decreased by $27.5 \pm 21.9 \%$. There were 19 (59\%) echocardiographic responders with reduction in LVESV of $\geq 15 \%$, which correlated with reduction in $\mathrm{LV}$ end-diastolic diameter $(r=0.6, P<.05$; 
TABLE 2: Difference in left ventricular function and dyssynchrony parameters in clinical responders versus nonresponders after long-term CRT.

\begin{tabular}{|c|c|c|c|}
\hline Variable & $\begin{array}{c}\text { Clinical responders }(n=24) \\
\text { Delta change 6-months after CRT }\end{array}$ & $\begin{array}{c}\text { Clinical nonresponders }(n=9) \\
\text { Delta change 6-months after CRT }\end{array}$ & $P$-value \\
\hline LV end-diastolic volume (mL) & $65 \pm 59$ & $12 \pm 15$ & .03 \\
\hline LV end-systolic volume (mL) & $74 \pm 59$ & $9 \pm 10$ & .008 \\
\hline LV ejection fraction (\%) & $15 \pm 8$ & $3 \pm 4$ & .001 \\
\hline LV end-diastolic diameter $(\mathrm{cm})$ & $0.5 \pm 0.1$ & $0.3 \pm 0.6$ & .6 \\
\hline LV fractional shortening (\%) & $0.05 \pm 0.1$ & $0.05 \pm 0.11$ & .9 \\
\hline TDI: Tf-Ts 6 basal segments (ms) & $34 \pm 34$ & $18 \pm 18$ & .24 \\
\hline TDI: Tf-Ts 12 segments (ms) & $13 \pm 12$ & $13 \pm 8$ & .96 \\
\hline TDI: septal-to-lateral delay (ms) & $28 \pm 26$ & $54 \pm 10$ & .07 \\
\hline 3D: SD of Tmsv in 6 basal segments (ms) & $51 \pm 33$ & $60 \pm 81$ & .70 \\
\hline 3D: SD of Tmsv in 12 segments (ms) & $16 \pm 13$ & $28 \pm 30$ & .16 \\
\hline 3D: SD of Tmsv in 16 segments (ms) & $34 \pm 35$ & $44 \pm 35$ & .48 \\
\hline 3D: SD of Tmsv in 12 segments as \% of R-R interval & $3.3 \pm 2.9 \%$ & $4.0 \pm 4.3 \%$ & .60 \\
\hline 3D: SD of Tmsv in 16 segments as \% of R-R interval & $4.4 \pm 4.0 \%$ & $3.5 \pm 5.4 \%$ & .59 \\
\hline
\end{tabular}

CRT: cardiac resynchronization therapy, LV: left ventricle, TDI: tissue Doppler imaging, Tf-Ts: difference in time to peak systolic velocity between the fastest and slowest LV segments, Tmsv: difference in longest and shortest time to minimal systolic volume in LV segments, SD: standard Deviation.

ROC $=0.56$ for LV diameter of 5 millimeters). As compared to the echocardiographic nonresponders, the responders had significant improvement in LV volumes, ejection fraction, and LV dimensions. Overall, there was significant improvement in dyssynchrony values after CRT (Table 1); however, the absolute delta changes in LV dyssynchrony values were similar in echocardiographic responders and nonresponders (Table 3). Echocardiographic responders were identified with $77 \%$ sensitivity and $34 \%$ specificity $(\mathrm{ROC}=0.67)$ with pre-CRT QRS duration of $150 \mathrm{~ms}$. Dyssynchrony score of $32 \mathrm{~ms}$ with TDI identified responders with a sensitivity of $73 \%$ and a specificity of $15 \%$. The echocardiographic response was not significantly different in those who met this cut-off value of dyssynchrony as compared to those who did not (Table 4).

4.4. Correlation between Dyssynchrony Indices by QRS duration, TDI, and 3D Volumetric Analysis. The pre-CRT QRS duration had significant correlation with dyssynchrony score by TDI in 12 segments $(r=0.7, P=.009)$ and with Tf-Ts in 6 basal segments $(r=0.6, P=.01)$. The dyssynchrony score by TDI in $12 \mathrm{LV}$ segments correlated with septallateral delay $(r=0.6, P<.05)$ and with Tf-Ts in 6 basal segments $(r=0.8, P<.05)$. Similarly, dyssynchrony scores by $3 \mathrm{D}$ volumetric analysis in $12 \mathrm{LV}$ segments and 16 segments expressed as percentage of cardiac cycle correlated significantly $(r=0.8, P<.05)$. However, there was no correlation between TDI and 3D measurements (Table 5).

\section{Discussion}

In our study, we noted improved LV systolic function and reduced LV volumes and dimensions after long-term CRT signifying reverse remodeling of LV. There was also reduction in LV dyssynchrony scores by several TDI and 3DTTE measurements after CRT; however, these did not help to identify responders before the procedure. The dyssynchrony scores by these techniques did not correlate with each other.

As seen in prior studies, there were more clinical responders than echocardiographic responders to CRT [6]. Subjective improvement in NYHA functional class was associated with objective improvement in 6-MWD as reported before $[1-3,23]$. The remaining patients with only clinical response without a reduction in LVESV probably had a placebo effect after pacemaker implantation or had some beneficial effect on LV mechanics without evident significant reverse remodeling. CRT was implanted in $5(13 \%)$ patients with NYHA functional class II symptoms, although their quality of life was significantly affected compared to their baseline and could be classified as IIIa, and opted to undergo CRT. The recent MADIT-CRT trial conducted in patients with mild heart failure symptoms showed a beneficial effect of CRT [24]. As reported in MADIT-CRT and in CAREHF trials [25], our study also showed similar clinical and echocardiographic response in ischemic and nonischemic etiology of cardiomyopathy [26].

Echocardiographic responders showed LV reverse remodeling without significant change in dyssynchrony as compared to nonresponders. Reverse remodeling of LV with improvement in LV volumes, dimensions, and LVEF with CRT has been reproduced in several studies $[3,8,24,27]$. LVESV is a reliable indicator of LV reverse remodeling, and it correlated with the clinical improvement in symptoms. However, pre-CRT LVESV did not identify CRT responders before implantation. The additional information on dyssynchrony by different methods did not seem to identify responders or improve the selection criteria. It is intuitive that correction of dyssynchrony is probably necessary to facilitate reverse remodeling and clinical improvement [28], but the available methods to 
TABLE 3: Difference in left ventricular function and dyssynchrony parameters in echocardiographic responders versus nonresponders after long-term CRT.

\begin{tabular}{|c|c|c|c|}
\hline \multirow[t]{2}{*}{ Variable } & $\begin{array}{l}\text { Echocardiographic responders } \\
\qquad(n=19)\end{array}$ & $\begin{array}{l}\text { Echocardiographic nonresponders } \\
\qquad(n=13)\end{array}$ & \multirow[t]{2}{*}{$P$-value } \\
\hline & Delta change 6-months after CRT & Delta change 6-months after CRT & \\
\hline LV end-diastolic volume $(\mathrm{mL})$ & $71 \pm 60$ & $18 \pm 26$ & .01 \\
\hline LV end-systolic volume (mL) & $82 \pm 58$ & $8 \pm 7$ & .0009 \\
\hline LV ejection fraction (\%) & $15 \pm 9$ & $6 \pm 7$ & .01 \\
\hline LV end-diastolic diameter $(\mathrm{cm})$ & $0.7 \pm 0.8$ & $0.03 \pm 0.6$ & .03 \\
\hline LV fractional shortening (\%) & $0.06 \pm 0.1$ & $0.03 \pm 0.07$ & .3 \\
\hline TDI: Tf-Ts 6 basal segments (ms) & $37 \pm 34$ & $15 \pm 17$ & .08 \\
\hline TDI: Tf-Ts 12 segments (ms) & $14 \pm 12$ & $10 \pm 8$ & .36 \\
\hline TDI: septal-to-lateral delay (ms) & $31 \pm 27$ & $41 \pm 24$ & .43 \\
\hline 3D: SD of Tmsv in 6 basal segments (ms) & $45 \pm 35$ & $68 \pm 68$ & .30 \\
\hline 3D: SD of Tmsv in 12 segments (ms) & $14 \pm 13$ & $27 \pm 25$ & .13 \\
\hline 3D: SD of Tmsv in 16 segments (ms) & $35 \pm 37$ & $42 \pm 33$ & .61 \\
\hline 3D: SD of Tmsv in 12 segments as \% of R-R interval & $3.5 \pm 3.0 \%$ & $3.5 \pm 4.0 \%$ & .97 \\
\hline 3D: SD of Tmsv in 16 segments as \% of R-R interval & $3.9 \pm 4.6 \%$ & $4.7 \pm 4.0 \%$ & .61 \\
\hline
\end{tabular}

${ }^{*} P<.05$, CRT: cardiac resynchronization therapy, LV: left ventricle, TDI: tissue Doppler imaging, Tf-Ts: difference in time to peak systolic velocity between the fastest and slowest LV segments, Tmsv: difference in longest and shortest time to minimal systolic volume in LV segments, SD: standard Deviation.

TABLE 4: Assessment of QRS duration and echocardiographic dyssynchrony parameters validated in other trials to predict long-term clinical and echocardiographic response.

\begin{tabular}{|c|c|c|c|c|c|}
\hline $\begin{array}{l}\text { Pre-CRT dyssynchrony } \\
\text { parameters }\end{array}$ & $\begin{array}{c}\text { Total } \\
(n=33)\end{array}$ & $\begin{array}{l}\text { Clinical } \\
\text { responders } \\
(n=24) \\
\end{array}$ & $P$-value & $\begin{array}{c}\text { Echocardiographic } \\
\text { responders } \\
(n=19) \\
\end{array}$ & $P$-value \\
\hline \multicolumn{6}{|l|}{ QRS (ms) } \\
\hline$<150$ & 9 & 6 & 0.6 & 6 & 0.7 \\
\hline$\geq 150$ & 24 & 18 & & 13 & \\
\hline \multicolumn{6}{|c|}{ TDI septal-lateral delay (ms) } \\
\hline$<65$ & 20 & 17 & 0.1 & 13 & 0.2 \\
\hline$\geq 65$ & 13 & 8 & & 6 & \\
\hline \multicolumn{6}{|c|}{ TDI (Tf-Ts) 12 LV segments (ms) } \\
\hline$<32$ & 6 & 5 & 0.5 & 4 & 0.6 \\
\hline$\geq 32$ & 27 & 19 & & 15 & \\
\hline \multicolumn{6}{|c|}{ TDI (Tf-Ts) 6 basal LV segments (ms) } \\
\hline$<83$ & 10 & 7 & 0.8 & 7 & 0.5 \\
\hline$\geq 83$ & 23 & 17 & & 12 & \\
\hline
\end{tabular}

CRT: cardiac resynchronization therapy, LV: left ventricle, TDI: tissue Doppler imaging, Tf-Ts: difference in time to peak systolic velocity between the fastest and slowest LV segments.

assess dyssynchrony are inadequate and time consuming. Until we have reliable techniques, QRS duration remains the simple choice to select patients for CRT. Although the cut-off value for selection of patients for CRT was $130 \mathrm{~ms}$, a cut-off value of $150 \mathrm{~ms}$ was used to identify responders as patients with wider QRS had better response to CRT in a large, randomized trial such as CARE-HF, and most of the patients enrolled had QRS $150 \mathrm{~ms}$ [1].

In our study, on applying the cut-off values based on prior trials, the responders did not show more dyssynchrony as compared to nonresponders prior to CRT $[8,9,11-$ 13, 29]. The PROSPECT trial evaluated seven TDI-based dyssynchrony scores and reported variable predictive values and concluded that no single echocardiographic measure was reliable in identifying responders. Interestingly, this study also highlighted the limitations of obtaining reliable images done at different centers [14], which is currently a problem in low-volume practices, not involved in research. Despite our study being conducted in a single university setting with a high volume of CRT insertions and echocardiographers with 
TABLE 5: Correlation between left ventricular dyssynchrony indices by QRS duration, TDI, and 3D volumetric analysis in patients undergoing CRT.

\begin{tabular}{|c|c|c|c|c|c|c|c|}
\hline & $\begin{array}{l}\text { Pearson's } \\
\text { correlation } \\
\text { coefficient }\end{array}$ & $\begin{array}{c}\text { QRS } \\
\text { dura- } \\
\text { tion } \\
(\mathrm{ms})\end{array}$ & $\begin{array}{c}\text { TDI: } \\
\text { septal-to- } \\
\text { lateral delay }\end{array}$ & $\begin{array}{l}\text { TDI: Tf-Ts } \\
6 \text { basal } \\
\text { segments } \\
(\mathrm{ms})\end{array}$ & $\begin{array}{c}\text { TDI: Tf-Ts } \\
12 \text { basal } \\
\text { segments } \\
\text { (ms) }\end{array}$ & $\begin{array}{l}\text { 3D: SD of Tmsv } \\
\text { in } 12 \text { segments } \\
\text { as } \% \text { of R-R } \\
\text { interval }\end{array}$ & $\begin{array}{c}\text { 3D: SD of Tmsv } \\
\text { in } 16 \text { segments } \\
\text { as \% of R-R } \\
\text { interval }\end{array}$ \\
\hline \multirow{2}{*}{ QRS duration (ms) } & Cor. coeff. & \multirow{2}{*}{1} & 0.3 & 0.6 & 0.7 & 0.05 & 0.1 \\
\hline & $P$-value & & 0.07 & $<0.05$ & $<0.05$ & 0.8 & 0.5 \\
\hline \multirow{2}{*}{ TDI: Septal-to-lateral delay } & Cor. coeff. & 0.3 & \multirow{2}{*}{1} & 0.6 & 0.6 & 0.06 & 0.03 \\
\hline & $P$-value & 0.07 & & $<0.05$ & $<0.05$ & 0.7 & 0.8 \\
\hline \multirow{2}{*}{$\begin{array}{l}\text { TDI: Tf-Ts } 6 \text { basal segments } \\
(\mathrm{ms})\end{array}$} & Cor. coeff. & 0.6 & 0.6 & \multirow{2}{*}{1} & 0.8 & 0.05 & 0.2 \\
\hline & $P$-value & $<0.05$ & $<0.05$ & & $<0.05$ & 0.7 & 0.2 \\
\hline \multirow{2}{*}{$\begin{array}{l}\text { TDI: Tf-Ts } 12 \text { basal segments } \\
(\mathrm{ms})\end{array}$} & Cor. coeff. & 0.7 & 0.6 & 0.8 & \multirow{2}{*}{1} & 0.03 & 0.1 \\
\hline & $P$-value & $<0.05$ & $<0.05$ & $<0.05$ & & 0.8 & 0.5 \\
\hline \multirow{2}{*}{$\begin{array}{l}\text { 3D: SD of Tmsv in } 12 \text { segments } \\
\text { as } \% \text { of } \mathrm{R}-\mathrm{R} \text { interval }\end{array}$} & Cor. coeff. & 0.05 & 0.06 & 0.05 & 0.03 & \multirow{2}{*}{1} & 0.8 \\
\hline & $P$-value & 0.8 & 0.7 & 0.7 & 0.8 & & $<0.05$ \\
\hline \multirow{2}{*}{$\begin{array}{l}3 \mathrm{D}: \mathrm{SD} \text { of Tmsv in } 16 \text { segments } \\
\text { as } \% \text { of } \mathrm{R}-\mathrm{R} \text { interval }\end{array}$} & Cor. coeff. & 0.1 & 0.03 & 0.2 & 0.1 & 0.8 & \multirow{2}{*}{1} \\
\hline & $P$-value & 0.5 & 0.8 & 0.2 & 0.5 & $<0.05$ & \\
\hline
\end{tabular}

CRT: cardiac resynchronization therapy, TDI: tissue Doppler imaging, Tf-Ts: difference in time to peak systolic velocity between the fastest and slowest LV segments, Tmsv: difference in longest and shortest time to minimal systolic volume in LV segments, SD: standard deviation, Cor. Coeff.: correlation coefficient.

an expertise in obtaining and interpreting TDI and 3D echo images, similar limitations were encountered.

A recent study by Marsan et al. showed good correlation in dyssynchrony scores by regional volumetric analysis by 3DTTE and gated myocardial perfusion single-photon emission computed tomography [17]. Kleijn et al. compared TDI and real-time 3DTTE and reported nonagreement between the two techniques when current dyssynchrony cut-off values were applied [21]. In our study, except for the QRS and TDI values, the TDI and 3DTTE dyssynchrony measurements did not correlate significantly, consistent with our observation in previous study [19]. The likely reason may be that TDI and 3DTTE measure different events like time-to-peak systolic velocity by TDI and time-to-minimal-systolic volume by 3DTTE, and these timings may not correlate, especially in dyssynchronous LV segments.

Early improvement in LV dyssynchrony has been shown to be predictive of long-term favorable response to CRT in prior studies $[26,27,30]$ unlike our study. But the goal is to identify the responders before a patient undergoes CRT procedure. Soliman et al. showed that 3DTTE dyssynchrony scores before CRT were useful in identifying long-term echocardiographic responders to CRT [20]. Multicenter studies are needed to see if this technique can reproduce similar results.

\section{Limitations}

The main limitation of our study is the small sample size, although studies with a larger sample such as PROSPECT yielded similar results. There are several other dyssynchrony parameters used in various studies, but we did not assess all of them. The median followup was limited to 7 months as the results of the study did not contribute to clinical decision making and were time consuming.

\section{Conclusion}

CRT leads to improved functional status, LV reverse remodeling, and improved synchrony between LV segments in patients with systolic HF. The dyssynchrony measurements by TDI and 3DTTE volumetric analysis do not correlate well with each other, and do not appear to be significantly related to measure of outcomes.

\section{Acknowledgments}

The authors would like to thank the staff in the echocardiography laboratory (Allan Paloma, Judy Chow and, Josephine Puryear) and the electrophysiology laboratory (Marcia Glassford, Kelly Green, and Linda Ottobani) for their invaluable support in conducting this study.

\section{References}

[1] J. G. F. Cleland, J. C. Daubert, E. Erdmann et al., "The effect of cardiac resynchronization on morbidity and mortality in heart failure," New England Journal of Medicine, vol. 352, no. 15, pp. 1539-1549, 2005.

[2] S. Cazeau, C. Leclercq, T. Lavergne et al., "Effects of multisite biventricular pacing in patients with heart failure and intraventricular conduction delay," New England Journal of Medicine, vol. 344, no. 12, pp. 873-880, 2001.

[3] W. T. Abraham, W. G. Fisher, A. L. Smith et al., "Cardiac resynchronization in chronic heart failure," New England Journal of Medicine, vol. 346, no. 24, pp. 1845-1853, 2002.

[4] G. Gregoratos, J. Abrams, A. E. Epstein et al., "ACC/AHA/ NASPE 2002 guideline update for implantation of cardiac pacemakers and antiarrhythmia devices: summary article. A report of the American College of Cardiology/American Heart Association Task Force on Practice Guidelines (ACC/AHA/NASPE Committee to Update the 1998 
Pacemaker Guidelines)," Journal of Cardiovascular Electrophysiology, vol. 13, no. 11, pp. 1183-1199, 2002.

[5] G. B. Bleeker, M. J. Schalij, S. G. Molhoek et al., "Relationship between QRS duration and left ventricular dyssynchrony in patients with end-stage heart failure," Journal of Cardiovascular Electrophysiology, vol. 15, no. 5, pp. 544-549, 2004.

[6] G. B. Bleeker, J. J. Bax, J. W. H. Fung et al., "Clinical versus echocardiographic parameters to assess response to cardiac resynchronization therapy," American Journal of Cardiology, vol. 97, no. 2, pp. 260-263, 2006.

[7] C. M. Yu, W. T. Abraham, J. Bax et al., "Predictors of response to cardiac resynchronization therapy (PROSPECT)—study design," American Heart Journal, vol. 149, no. 4, pp. 600-605, 2005.

[8] P. Sogaard, H. Egeblad, W. Y. Kim et al., "Tissue Doppler imaging predicts improved systolic performance and reversed left ventricular remodeling during long-term cardiac resynchronization therapy," Journal of the American College of Cardiology, vol. 40, no. 4, pp. 723-730, 2002.

[9] M. V. Pitzalis, M. Iacoviello, R. Romito et al., "Cardiac resynchronization therapy tailored by echocardiographic evaluation of ventricular asynchrony," Journal of the American College of Cardiology, vol. 40, no. 9, pp. 1615-1622, 2002.

[10] M. V. Pitzalis, M. Iacoviello, R. Romito et al., "Ventricular asynchrony predicts a better outcome in patients with chronic heart failure receiving cardiac resynchronization therapy," Journal of the American College of Cardiology, vol. 45, no. 1, pp. 65-69, 2005.

[11] J. J. Bax, G. B. Bleeker, T. H. Marwick et al., "Left ventricular dyssynchrony predicts response and prognosis after cardiac resynchronization therapy," Journal of the American College of Cardiology, vol. 44, no. 9, pp. 1834-1840, 2004.

[12] D. Notabartolo, J. D. Merlino, A. L. Smith et al., "Usefulness of the peak velocity difference by tissue Doppler imaging technique as an effective predictor of response to cardiac resynchronization therapy," American Journal of Cardiology, vol. 94, no. 6, pp. 817-820, 2004.

[13] C. M. Yu, W. H. Fung, H. Lin, Q. Zhang, J. E. Sanderson, and C. P. Lau, "Predictors of left ventricular reverse remodeling after cardiac resynchronization therapy for heart failure secondary to idiopathic dilated or ischemic cardiomyopathy," American Journal of Cardiology, vol. 91, no. 6, pp. 684-688, 2003.

[14] E. S. Chung, A. R. Leon, L. Tavazzi et al., "Results of the predictors of response to crt (prospect) trial," Circulation, vol. 117, no. 20, pp. 2608-2616, 2008.

[15] O. I. I. Soliman, D. A. M. J. Theuns, M. L. Geleijnse et al., "Spectral pulsed-wave tissue Doppler imaging lateral-to-septal delay fails to predict clinical or echocardiographic outcome after cardiac resynchronization therapy," Europace, vol. 9, no. 2, pp. 113-118, 2007.

[16] Q. Zhang, C. M. Yu, J. W. H. Fung et al., "Assessment of the effect of cardiac resynchronization therapy on intraventricular mechanical synchronicity by regional volumetric changes," American Journal of Cardiology, vol. 95, no. 1, pp. 126-129, 2005.

[17] N. A. Marsan, M. M. Henneman, J. Chen et al., "Real-time three-dimensional echocardiography as a novel approach to quantify left ventricular dyssynchrony: a comparison study with phase analysis of gated myocardial perfusion single photon emission computed tomography," Journal of the American Society of Echocardiography, vol. 21, no. 7, pp. 801-807, 2008.
[18] S. Kapetanakis, M. T. Kearney, A. Siva, N. Gall, M. Cooklin, and M. J. Monaghan, "Real-time three-dimensional echocardiography: a novel technique to quantify global left ventricular mechanical dyssynchrony," Circulation, vol. 112, no. 7, pp. 992-1000, 2005.

[19] S. S. Kuppahally, M. B. Fowler, R. Vagelos et al., "Worsening of left ventricular end-systolic volume and mitral regurgitation without increase in left ventricular dyssynchrony on acute interruption of cardiac resynchronization therapy," Echocardiography, vol. 26, no. 7, pp. 759-765, 2009.

[20] O. I. I. Soliman, M. L. Geleijnse, D. A. M. J. Theuns et al., "Usefulness of left ventricular systolic dyssynchrony by real-time three-dimensional echocardiography to predict long-term response to cardiac resynchronization therapy," American Journal of Cardiology, vol. 103, no. 11, pp. 15861591, 2009.

[21] S. A. Kleijn, J. van Dijk, C. C. de Cock, C. P. Allaart, A. C. van Rossum, and O. Kamp, "Assessment of intraventricular mechanical dyssynchrony and prediction of response to cardiac resynchronization therapy: comparison between tissue Doppler imaging and real-time three-dimensional echocardiography," Journal of the American Society of Echocardiography, vol. 22, no. 9, pp. 1047-1054, 2009.

[22] J. D. Thomas, "How leaky is that mitral valve? Simplified Doppler methods to measure regurgitant orifice area," Circulation, vol. 95, no. 3, pp. 548-550, 1997.

[23] J. B. Young, W. T. Abraham, A. L. Smith et al., "Combined cardiac resynchronization and implantable cardioversion defibrillation in advanced chronic heart failure: the MIRACLE ICD trial," Journal of the American Medical Association, vol. 289, no. 20, pp. 2685-2694, 2003.

[24] A. J. Moss, W. J. Hall, D. S. Cannom et al., "Cardiacresynchronization therapy for the prevention of heart-failure events," New England Journal of Medicine, vol. 361, no. 14, pp. 1329-1338, 2009.

[25] G. Wikstrom, C. Blomström-Lundqvist, B. Andren et al., "The effects of aetiology on outcome in patients treated with cardiac resynchronization therapy in the CARE-HF trial," European Heart Journal, vol. 30, no. 7, pp. 782-788, 2009.

[26] N. A. Marsan, G. B. Bleeker, R. J. van Bommel et al., "Comparison of time course of response to cardiac resynchronization therapy in patients with ischemic versus nonischemic cardiomyopathy," American Journal of Cardiology, vol. 103, no. 5, pp. 690-694, 2009.

[27] A. J. Bank, C. L. Kaufman, A. S. Kelly et al., "Results of the prospective Minnesota study of ECHO/TDI in cardiac resynchronization therapy (PROMISE-CRT) study," Journal of Cardiac Failure, vol. 15, no. 5, pp. 401-409, 2009.

[28] G. B. Bleeker, S. A. Mollema, E. R. Holman et al., "Left ventricular resynchronization is mandatory for response to cardiac resynchronization therapy: analysis in patients with echocardiographic evidence of left ventricular dyssynchrony at baseline," Circulation, vol. 116, no. 13, pp. 1440-1448, 2007.

[29] C.-M. Yu, H. Lin, Q. Zhang, and J. E. Sanderson, "High prevalence of left ventricular systolic and diastolic asynchrony in patients with congestive heart failure and normal QRS duration," Heart, vol. 89, no. 1, pp. 54-60, 2003.

[30] R. Abdelhadi, E. Adelstein, A. Voigt, J. Gorcsan, and S. Saba, "Measures of left ventricular dyssynchrony and the correlation to clinical and echocardiographic response after cardiac resynchronization therapy," American Journal of Cardiology, vol. 102, no. 5, pp. 598-601, 2008. 


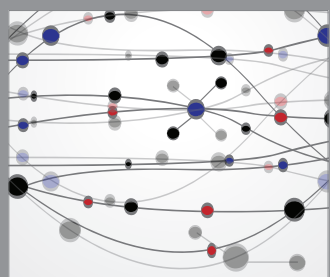

The Scientific World Journal
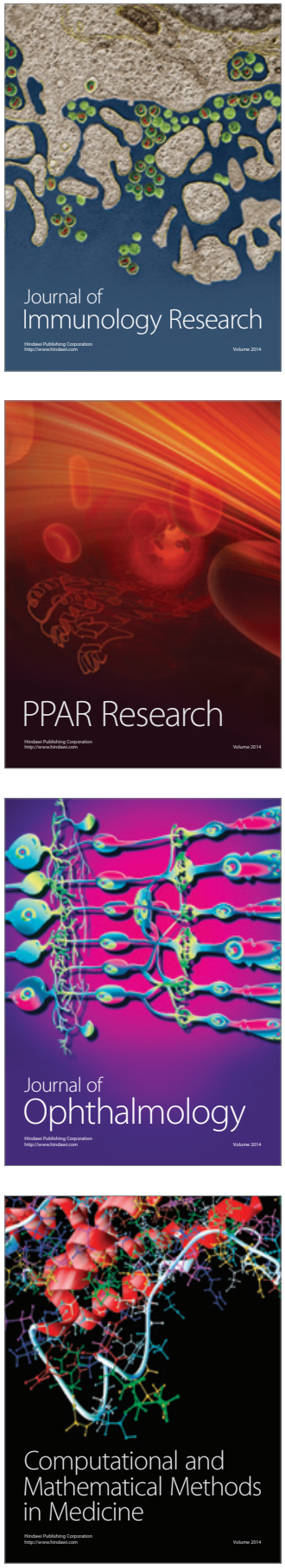

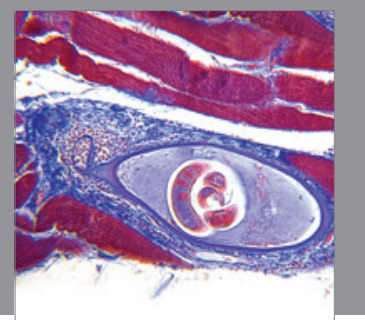

Gastroenterology

Research and Practice
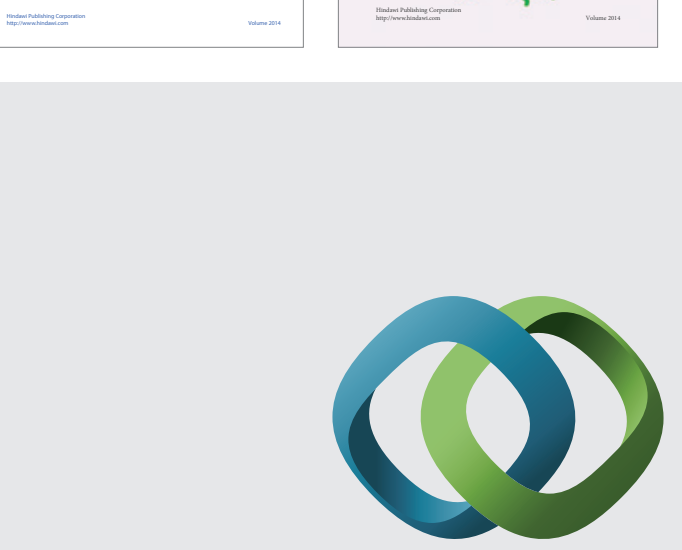

\section{Hindawi}

Submit your manuscripts at

http://www.hindawi.com
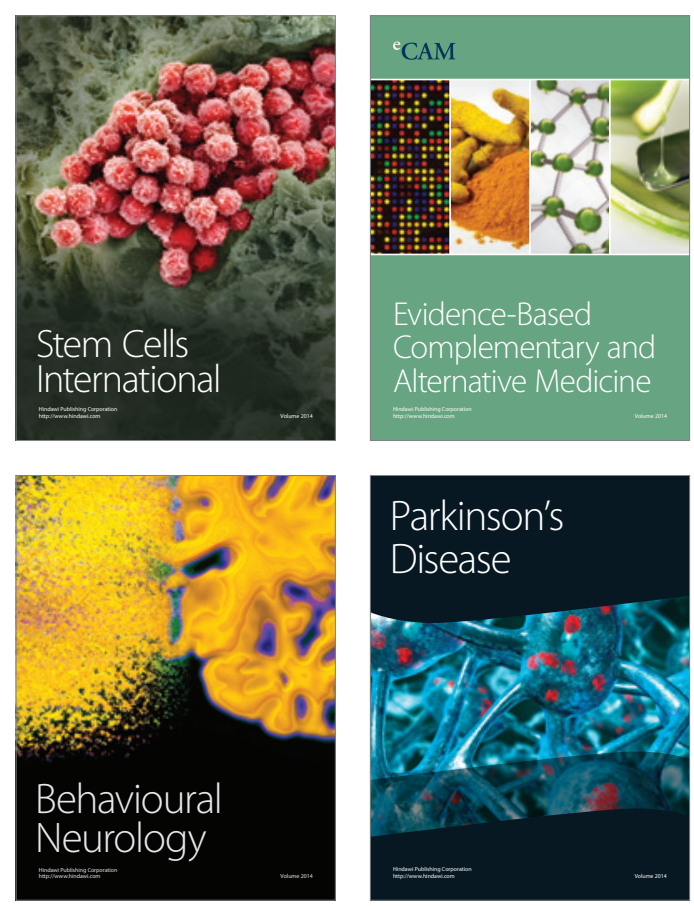

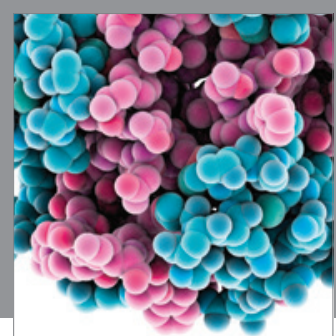

Journal of
Diabetes Research

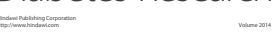

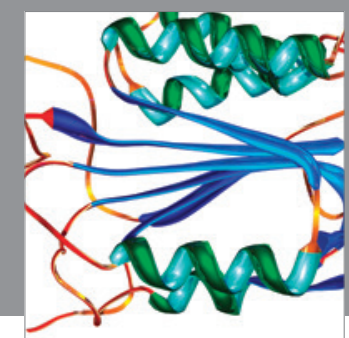

Disease Markers
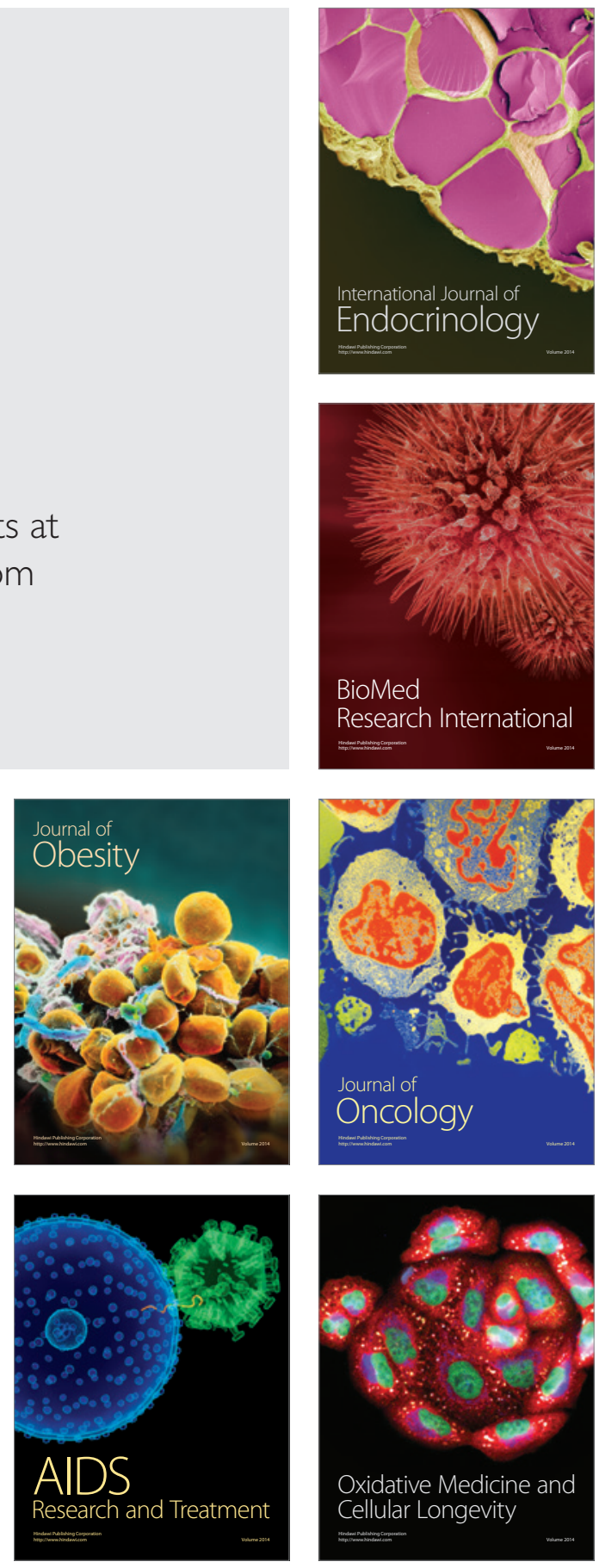